\title{
MELAS or more
}

\section{Melas}

With interest we read the review by Lorenzoni et al. about the diagnostic criteria of mitochondrial encephalopathy lactic acidosis and stroke-like episodes (MELAS) syndrome ${ }^{1}$. We have the following comments, and concerns.

Concerning the cardiac involvement it is essential to be more specific with the variable manifestations, since different manifestations require different treatment and have a variable outcome. The m.3243A > G mutation has been reported in association with dilated cardiomyopathy ${ }^{2}$ and hypertrophic cardiomyopathy. The MELAS phenotype has been reported in association with dilated cardiomyopathy ${ }^{3}$ and hypertrophic cardiomyopathy ${ }^{4,5}$. The MELAS phenotype or the m.3243A > G mutation have not been described in association with restrictive cardiomyopathy, noncompaction, or Takotsubo-syndrome. Arrhythmias have been hardly described in MELAS syndrome but conduction defects such as pre-excitation (Wolff-Parkinson-White syndrome), AV-conduction defects ${ }^{7}$, or intra-ventricular conduction defects $^{8}$ were reported. The most frequent of the conduction defects in MELAS is Wolff-Parkinson-White syndrome. In a single patient sudden cardiac death has been described ${ }^{9}$.

Concerning the pathogenesis of stroke-like-episodes it has been also hypothesized that an initial seizure may cause stress in affected neurons resulting in secondary metabolic break-down clinically manifesting as stroke-like-episode ${ }^{10}$. An argument for the seizure hypothesis is that stroke-like episodes are frequently associated with seizures and that appropriate antiepileptic treatment may be beneficial also for stroke-like-episodes.

Though the authors mention that epilepsy as an "absolute criterion" for diagnosing MELAS they do not mention the antiepileptic treatment, which is critical in patients with mitochondrial disorders (MIDs) since mitochondrion-toxic antiepileptic drugs should be avoided. Particularly mitochondrion-toxic are valproic acid, carbamazepine, phenobarbital, and phenytoin. Less mitochondrion-toxic are ethosuximide, oxcarbazepine, topiramate, felbamate, zonisamide, lamotrigine, levetirazetam, and gabapentine. However, mitochondrion-toxicity of antiepileptic drugs may depend on the mutation load within a tissue, which means that the toxicity increases with the amount of heteroplasmy. If liver or kidneys are additionally involved in MELAS, metabolisation of antiepileptic drugs may be further impaired and may contribute to the toxicity of these agents towards mitochondria.

The review lacks description of lactate-stress-tests as appropriate tools for suspecting a MID. Particularly, the lactate-stress-test on a cycle ergometer can be helpful to direct the further diagnostic work-up in these patients ${ }^{11}$. The principle of the test relies on the finding that patients with a MID may show a significant increase of serum lactate already at a minimal constant work-load below the lactate threshold ${ }^{12}$. Though the test can produce false positive and false negative results, it can help to decide if further invasive diagnostic steps should be initiated.

Overall, this excellent review could widen the spectrum of discussion by inclusion of issues addressed above. Since MELAS is a multisystem disease it requires a multi-professional therapeutic approach. Since particularly brain and heart functions determine the outcome of these patients it is important that neurologists closely work together with cardiologists. Some of the clinical cerebral or cardiac manifestations are particularly accessible to treatment why it is essential that patients receive appropriate treatment in due time.

Josef Finsterer ${ }^{1}$, Marlies Frank ${ }^{2}$

\section{References}

1. Lorenzoni PJ, Werneck LC, Kay CS, Silvado CE, Scola RH. When should MELAS (Mitochondrial myopathy, Encephalopathy, Lactic Acidosis, and Stroke-like episodes) be the diagnosis? Arq Neuropsiquiatr. 2015;73(11):959-67. doi:10.1590/0004-282X20150154
2. Stalder N, Yarol N, Tozzi P, Rotman S, Morris M, Fellmann F et al. Mitochondrial A3243G mutation with manifestation of acute dilated cardiomyopathy. Circ Heart Fail. 2012;5(1):e1-3. doi:10.1161/CIRCHEARTFAILURE.111.963900 
3. Cristofari M, Bertocchi P, Viganò M. The MELAS syndrome and dilated-hypertrophic cardiomyopathy: a case report. G Ital Cardiol. 1995;25(1):69-76. Italian.

4. Wortmann SB, Champion MP, Heuvel L, Barth H, Trutnau B, Craig K et al.. Mitochondrial DNA m.3242G > A mutation, an under diagnosed cause of hypertrophic cardiomyopathy and renal tubular dysfunction? Eur J Med Genet. 2012;55(10):552-6. doi:10.1016/j.ejmg.2012.06.002

5. Menotti F, Brega A, Diegoli M, Grasso M, Modena MG, Arbustini E. A novel mtDNA point mutation in tRNA(Val) is associated with hypertrophic cardiomyopathy and MELAS. Ital Heart J. 2004;5(6):460-5.

6. Poretti A, Balmer C. Wolff-Parkinson-White syndrome in a child with recurrent seizures. Cardiol Young. 2010;20(1):78-9. doi:10.1017/S1047951109991004

7. Hattori Y, Matsuda M, Eizawa T, Nakajima K. A case of mitochondrial myopathy, encephalopathy, lactic acidosis and stroke-like episodes (MELAS), showing temporary improvement during the treatment with eicosapentaenoic acid ethyl ester. Rinsho Shinkeigaku. 2001;41(10):668-72. Japanese.

8. Reato S, Spartà S, D’Este D. Intraventricular conduction disturbances and paroxysmal atrioventricular block in a young patient with MELAS.J Cardiovasc Med (Hagerstown). 2015;16(Suppl 2):S100-3. doi:10.2459/JCM.0b013e3283410351

9. Taniguchi A, Kitagawa T, Kuzuhara S. MELAS sudden death due to paroxysmal arrhythmia. Nihon Rinsho. 2002;60 Suppl 4:606-9. Japanese.

10. lizuka T, Sakai F, Suzuki N, Hata T, Tsukahara S, Fukuda M, Takiyama Y. Neuronal hyperexcitability in stroke-like episodes of MELAS syndrome. Neurology. 2002;59(6):816-24. doi:10.1212/WNL.59.6.816

11. Finsterer J, Milvay E. Lactate stress testing in 155 patients with mitochondriopathy. Can J Neurol Sci. 2002;29(1):49-53. doi:10.1017/S0317167100001712

12. Finsterer J, Shorny S, Capek J, Cerny-Zacharias C, Pelzl $\mathrm{B}$, Messner R et al.. Lactate stress test in the diagnosis of mitochondrial myopathy.J Neurol Sci. 1998;159(2):176-80. doi:10.1016/S0022-510X(98)00170-1 